\title{
LA BIOTECNOLOGÍA, LA LUCHA CONTRA EL CAMBIO CLIMÁTICO Y LOS BIOCARBURANTES
}

\author{
ISABEL HERNÁNDEZ SAN JUAN \\ Doctorado en Derecho \\ Universidad Carlos III de Madrid \\ ihernand@der-pu.uc3m.es
}

Recibido: 15 de octubre de 2014 / Aceptado: 13 de noviembre de 2014

RESUMEN: La energía se agota y los biocarburantes son, o han sido hasta ahora, una de las formas de energía alternativa exitosas para combatir el cambio climático. Al mismo tiempo, la investigación biotecnológica que creaba y avalaba esta nueva forma de producción energética ha sido ampliamente apoyada y desarrollada por instituciones y empresas dada su versatilidad y utilidad en otros campos como el de la salud y la lucha contra la contaminación o la protección del medio ambiente, entre otros aspectos beneficiosos para la sociedad. Sin embargo, en el caso de la biotecnología, ésta presenta serios rasgos de incertidumbre científica en relación con la energía y la seguridad alimentaria, pero también con la agricultura. Dadas estas cuestiones problemáticas, se sugiere cuestionar la formulación actual de las políticas en las que se articulan todas estas actividades; en esa tarea de eventual reformulación están implicados el desarrollo de distintos cursos de acción (alternativas, propuestas, opciones) aceptables y pertinentes para enfrentarse a los problemas públicos, pues las políticas deben concebirse como soluciones a los problemas públicos.

RESUM: L'energia s'exhaureix i els biocarburants són, o han estat fins ara, una de les formes d'energia alternativa exitoses per combatre el canvi climàtic. Al mateix temps, la investigació biotecnològica que creava i avalava aquesta nova forma de producció energètica ha rebut un ampli suport $i$ ha estat desenvolupava per institucions i empreses, atesa la seva versatilitat i utilitat en altres camps com el de la salut i la lluita contra la contaminació o la protecció del medi ambient, entre altres aspectes beneficiosos per a la 
societat. Tanmateix, en el cas de la biotecnologia, aquesta presenta seriosos trets d'incertesa científica en relació amb l'energia i la seguretat alimentàries, però també amb l'agricultura ateses aquestes qüestions problemàtiques, es suggereix qüestionar la formulació actual de les polítiques en les que s'articulen totes aquestes activitats; en aquesta tasca de reformulació eventual estan implicats el desenvolupament de diferents cursos d'acció (alternatives, propostes, opcions) acceptables i pertinents per enfrontar-se als problemes públics, ja que les polítiques han de concebre's com a solució per aquests.

ABSTRACT: The energy is depleted and biofuels have been so far, one kind of alternative and renewable energy successful to tackling climate change. While biotechnology research that created this new kind of energy production has been widely supported and developed by institutions and companies given its versatility and extremely useful in other fields such as health, combating pollution or protection of the environment, among other beneficial effects for society. However biotechnology presents serious scientific uncertainty in relation to energy, food security, and agriculture. Given these problematic issues this case study suggests asking about the current formulation of sectoral policies in which these activities are organized; in the task of further refinement are involved different courses of action (alternatives, proposals, options) acceptable and relevant to deal public problems, then policies must be conceived as solutions to them.

PALABRAS CLAVE: Biotecnología - Cambio climático - Biocarburantes Políticas sectoriales de la Unión Europea.

PARAULES CLAU: Biotecnologia - Canvi climàtic - Biocarburants - Polítiques sectorials de la Unió Europea.

KEYWORDS: Biotechnology - Climate change - Biofuels - EU policies.

SUMARIO: I. Introducción. II. Breve análisis de las políticas implicadas y competencias para su desarrollo. 1. Investigación y desarrollo tecnológico. 2. La biotecnología. 3. Medio ambiente y cambio 
climático. 4. Energía y biocarburantes. 5. Política Agrícola Común (PAC) e industria agroalimentaria. III. A modo de conclusión. IV. Bibliografía.

\section{INTRODUCCIÓN}

Al reflexionar sobre los tres términos del título del presente estudio, es necesario primero explicar los aspectos que confluyen y coinciden en ellos y cuáles son los que pueden llegar a disgregar la aparente feliz unión en este enunciado de "trío ganador"”. La biotecnología y los biocarburantes tienen en común ser modernas aplicaciones tecnológicas que persiguen distintas finalidades - entre otras, la lucha contra el cambio climático-, derivándose de la política sectorial común europea en materia industrial, de investigación y desarrollo tecnológico, si bien participan en otras políticas sectoriales como, por ejemplo, la energética (en el caso de los biocarburantes) o en la política agrícola, la protección del medio ambiente o la salud de las personas (en el caso de la biotecnología).

Al mismo tiempo, otras políticas sectoriales como la de protección del medio ambiente y la Política Agrícola Común (PAC) están conectadas con el cambio climático. Todo ello conlleva una gran complejidad para la propia ejecución de las políticas de forma real y efectiva. En resumen, las políticas sectoriales de la Unión Europea (UE) implicadas en el presente artículo se reflejan en el siguiente cuadro:

\footnotetext{
${ }^{1}$ Este artículo es el resultado de la contribución de la autora al congreso 12th IUCNAEL Colloquium 2014 "Energy for a fair society in a safe planet", celebrado en Tarragona del 30 de junio al 5 de julio de 2014.
} 


\begin{tabular}{|c|c|c|c|}
\hline $\begin{array}{c}\text { Política industrial, } \\
\text { de investigación y } \\
\text { desarrollo } \\
\text { tecnológico }\end{array}$ & $\begin{array}{c}\text { Política de medio } \\
\text { ambiente }\end{array}$ & Política energética & $\begin{array}{c}\text { PAC } \\
\text { (Política Agrícola } \\
\text { Común) }\end{array}$ \\
\hline Biotecnología & Biotecnología & Biotecnología & Biotecnología \\
\hline Biocarburantes & Biocarburantes & Biocarburantes & Biocarburantes \\
\hline Cambio climático & Cambio climático & Cambio climático & Cambio climático \\
\hline
\end{tabular}

En este sentido, no hay que olvidar que la lucha contra el cambio climático es un elemento fundamental del programa medioambiental de la UE y se ha integrado cada vez más en otras políticas como la energética, la de transportes, la agrícola o la de desarrollo regional. La protección del medio ambiente como objetivo y política “clásicos" en el derecho europeo y en el acervo comunitario contempla ahora de manera esencial la lucha contra el cambio climático. Muy resumidamente, el objetivo de la política climática de la UE es limitar el calentamiento del planeta a $2{ }^{\circ} \mathrm{C}$ sobre $\operatorname{los}$ niveles térmicos medios de la era preindustrial. La UE se ha comprometido a reducir, hasta el año 2020, las emisiones de gases de efecto invernadero al menos un $20 \%$ respecto a los niveles de 1990, a mejorar la eficiencia energética en un $20 \%$ y a aumentar el porcentaje de las energías renovables hasta alcanzar el $20 \%$ del consumo final.

Recientemente esta posición ha cambiado y el Consejo Europeo ha determinado el siguiente objetivo actualizado de reducción de emisiones de gases de efecto invernadero:

El Consejo Europeo ha refrendado el objetivo, vinculante para la UE, de reducir las emisiones de gases de efecto invernadero de la Unión por lo menos en un $40 \%$ para 2030 con respecto a los valores de 1990. A tal fin: la UE cumplirá colectivamente el objetivo de la manera más eficaz posible en términos de coste, con reducciones en los sectores sujetos y no sujetos al régimen de comercio de derechos de emisión del $43 \%$ y del $30 \%$, respectivamente, en 2030 en comparación con 2005; todos los 
Estados miembros participarán en este esfuerzo, conciliando consideraciones de equidad y solidaridad ${ }^{2}$.

En definitiva, todas las acciones o políticas sectoriales recogidas en el cuadro anterior son líneas de actuación que la UE decide seguir en determinados ámbitos que afectan directamente a la vida de los ciudadanos y de las empresas y que se proponen lograr los objetivos generales que se ha fijado la misma UE. Implementar en la práctica y ejecutar todas estas políticas al mismo tiempo de forma coordinada ha sido y es un reto ambicioso que requiere unidad de acción, pues garantiza un efecto de palanca que permite obtener mejores resultados. En su andadura, la UE ha desarrollado una serie de políticas complementarias del mercado único en relación con los ámbitos más diversos y con un grado variable de armonización: desde verdaderas políticas comunes hasta simples medidas de cooperación ${ }^{3}$. Sin olvidar la perspectiva internacional, pues, siendo un tema de interés global o más amplio que el regional europeo, no se tiene una determinación clara de los responsables de la regulación en este nivel, o dicho de otro modo, habría que interrogarse si ciertamente existen responsables globales.

Y, por último, cabe recordar que en todos los sectores de los que aquí se habla la Dirección General de Asuntos Económicos y Financieros de la UE apoya el desarrollo de políticas rentables, esto es, que alcancen sus objetivos al mínimo coste para la sociedad al mismo tiempo que respaldan el desarrollo sostenible y estimulan el crecimiento económico y el empleo. Para lograr esto, dicha Dirección General proporciona análisis económicos que sirven de base a la elaboración de políticas económicas y alienta a otras direcciones generales a buscar orientaciones que permitan alcanzar los resultados previstos mejorando el funcionamiento del mercado, sin colocar obstáculos innecesarios en su camino ${ }^{4}$.

\footnotetext{
2 Conclusiones del Consejo Europeo (23 y 24 de octubre de 2014). Accesible en $<$ http://www.consilium.europa.eu/uedocs/cms_data/docs/pressdata/es/ec/145425.pdf $>$ Fecha de consulta 19 de noviembre de 2014.

${ }^{3}$ Políticas sectoriales de la Unión Europea como la de cohesión, agricultura, pesca, medio ambiente, sanidad, derechos de los consumidores, transporte, turismo, energía, industria, investigación, empleo, asilo e inmigración, así como fiscalidad, justicia, cultura, educación y deporte.

${ }^{4}$ Función de la Dirección General de Asuntos Económicos y Financieros. Toda la información en $<$ http://ec.europa.eu/economy_finance/structural_reforms/sectoral/index_en.htm>.
} 


\section{BREVE ANÁLISIS DE LAS POLÍTICAS IMPLICADAS Y COMPETENCIAS PARA SU DESARROLLO}

En el plano competencial, los tratados prevén estas actividades/políticas. El Tratado de Funcionamiento de la Unión Europea (TFUE), dentro del título I sobre categorías y ámbitos de competencias de la Unión, señala en su artículo 4.2 que las competencias compartidas entre la Unión y los Estados miembros se aplicarán a los siguientes ámbitos principales:

Las competencias compartidas entre la Unión y los Estados miembros se aplicarán a los siguientes ámbitos principales: el mercado interior; la política social, en los aspectos definidos en el presente Tratado; la cohesión económica, social y territorial; la agricultura y la pesca, con exclusión de la conservación de los recursos biológicos marinos; el medio ambiente; la protección de los consumidores; los transportes; las redes transeuropeas; la energía; el espacio de libertad, seguridad y justicia; los asuntos comunes de seguridad en materia de salud pública, en los aspectos definidos en el presente Tratado.

Y en el apartado tercero, el artículo 4 dice:

En los ámbitos de la investigación, el desarrollo tecnológico y el espacio, la Unión dispondrá de competencia para llevar a cabo acciones, en particular destinadas a definir y realizar programas, sin que el ejercicio de esta competencia pueda tener por efecto impedir a los Estados miembros ejercer la suya.

Veamos por separado las que atañen a este estudio.

\section{Investigación y desarrollo tecnológico}

Se dice que ha comenzado una nueva etapa en la investigación e innovación comunitarias. El Parlamento Europeo ha aprobado el nuevo marco por el que se regirá la $\mathrm{I}+\mathrm{D}+\mathrm{i}$ de la UE durante los próximos años: Horizon 2020, que sustituirá al anterior VII Programa Marco, siendo el mayor programa de investigación e innovación de la UE aprobado con casi 80.000 millones de euros de fondos disponibles (2014-2020), además de la inversión privada que este dinero va a atraer. Promete más avances, 
descubrimientos y primicias mundiales mediante la adopción de grandes ideas que irán del laboratorio al mercado: "from lab to market"

La importancia que Horizon 2020 concede a la biotecnología es indudable, pues la considera como "una de las seis tecnologías industriales clave para favorecer el liderazgo industrial de la Unión Europea”, junto con las TIC, la nanotecnología, los materiales avanzados, la investigación espacial y el proceso y la fabricación avanzados. Pero dentro de Horizon las áreas son amplias y diversas y, además de la biotecnología, se encuentra la energía; para nuestro estudio, nos interesa el apartado de las tecnologías bajas en carbón configuradas como reto social dentro del programa. Es importante desarrollar y llevar al mercado soluciones tecnológicas asequibles, rentables y eficientes de los recursos para descarbonizar el sistema energético de manera sostenible, garantizar un suministro de energía seguro y completar el mercado interior de la energía. Las actividades de investigación dentro de este eje se centrarán en la energía fotovoltaica, la energía solar concentrada, la energía del viento, la calefacción y refrigeración renovables, el almacenamiento de energía, los biocombustibles y los combustibles alternativos, la captura y el almacenamiento de carbono, etc.

En la web española encargada de ofrecer a nuestro país este gran programa de investigación, se dice en similar sentido que la "energía segura, limpia y eficiente" es la tercera prioridad temática de los retos sociales planteados en Horizonte $2020^{6}$. De los tres pilares dedicados a la investigación - liderazgo industrial, retos sociales y ciencia excelente-, las líneas generales de la actividad en los procesos industriales basados en la biotecnología son: capacitar a la industria europea, entre otros muchos, en los sectores energético y de la elaboración de alimentos para desarrollar nuevos productos y procesos que satisfagan las demandas industriales y sociales; crear alternativas competitivas y mejoradas basadas en la biotecnología para sustituir las que ya existen; y aprovechar el potencial de la biotecnología para detectar, controlar, prevenir y eliminar la contaminación.

\footnotetext{
${ }^{5}$ Información accesible en <http://ec.europa.eu/programmes/horizon2020/en/what-horizon-2020> Debe estudiarse con cautela la expresión y lo que comporta, pues recuerda a la de otra política como es la de la seguridad alimentaria con su archiconocida frase "de la granja a la mesa": "from farm to fork". A pesar de todas las medidas existentes de protección de la larga cadena alimentaria y del pretendido control, hoy día siguen produciéndose graves crisis y fraudes alimentarios.

${ }^{6}$ Programa Marco de Investigación e Innovación de la Unión Europea (2014-2020). El presupuesto global establecido para el período 2014-2020 es de 5.931 millones de euros.
} 
Grosso modo, todos estos recursos económicos destinados en general a la creación de sociedades mejores y más avanzadas gracias a la investigación se encuentran con la dificultad en el plano competencial de una delicada separación y clarificación de los campos a los que ésta se dedica. Es decir, en lo que respecta a las competencias europeas en investigación científica, hay que señalar, como hace ELVIRA, lo siguiente: "[...] nos encontramos con una normativa dispersa, vinculada a las competencias comunitarias, con un diferente carácter, precisamente, en virtud del tipo de competencia; de esta forma con frecuencia es difícil expurgar los aspectos que pueden afectar a la investigación del resto de las competencias de las que se derivan [...]. En el TFUE - fuera de la Carta de Derechos Fundamentales de la UE- se encuentran competencias compartidas que afectan o pueden afectar a la investigación científica, en particular el Título XIV de la Tercera Parte, dedicado a la Salud Pública”, en particular el artículo 168. Junto a estas medidas, otras también pueden, en su caso, incidir en las materias, como la protección de los consumidores (tercera parte, título XV), el medio ambiente (tercera parte, título XX), la política comercial común (quinta parte, título II) y, claro está, la investigación y el desarrollo tecnológico (tercera parte, título XIX), donde, entre otros aspectos, se establece que "la Unión y sus Estados miembros coordinarán su acción en materia de investigación y de desarrollo tecnológico, con el fin de garantizar la coherencia recíproca de las políticas nacionales y de las políticas de la Unión" (art. 181.1 TFUE) ${ }^{7}$.

\section{La biotecnología}

La biotecnología ofrece diversas y atractivas alternativas para producir energía y para mejorar la eficiencia en su utilización a la vez que valoriza residuos urbanos y forestales y subproductos de determinadas industrias, reduciendo su impacto ambiental. Algunos procesos biotecnológicos para la producción de energía pueden considerarse "clásicos" dentro del estado del arte, como puede ser la producción de biogás a partir de aguas residuales, mientras que otros son objeto de investigación en la actualidad ${ }^{8}$.

\footnotetext{
${ }^{7}$ ELVIRA, A., "El derecho a la investigación científica en el marco de la Unión Europea”, Chueca, R. (dir.), La investigación científica como derecho fundamental, Comares, 2012, pp. 69-100 y pp. 72 y ss.

${ }^{8}$ MERCEDES BALLESTEROS: jefa Unidad de Procesos Biotecnológicos para la Producción de Energía/JAVIER DUFOUR: jefe Unidad de Análisis de Sistemas/FÉLIX MARÍN: responsable de Desarrollo y Transferencia de Tecnología del Instituto IMDEA Energía. Consultado el 14 de junio de 2014: <http://www.madrimasd.org/informacionIdi/analisis/opinion/opinion.asp?id=59998>
} 
Desde la UE han sido muchos los esfuerzos para lograr que la biotecnología y las ciencias de la vida tuvieran un amplio y extenso desarrollo en el territorio. Como aplicación práctica y palpable que evidencia esta estrategia biotecnológica se encuentra la creación de energía alternativa con materiales de procedencia vegetal. Las principales aplicaciones de la biotecnología blanca o industrial son para la producción de energía, la biorremediación y la biodetergencia:

\begin{abstract}
"Los materiales de procedencia vegetal pueden proporcionar tanto los elementos moleculares básicos como moléculas más complejas para el sector energético. [...] Con las condiciones económicas y fiscales adecuadas, la biomasa podría contribuir a la creación de energía alternativa, con carburantes biológicos sólidos y líquidos como el biodiesel y el bioetanol y procesos como la biodesulfatación. También se recurre a la biotecnología para seleccionar o mejorar, las características específicas de algunos organismos. [...] Plantas modificadas genéticamente utilizadas en sectores como el de la producción de biocarburantes o del papel se interesarán por plantas que ofrezcan un mayor rendimiento"
\end{abstract}

En la medida en que se aplican diferentes niveles de competencia, la estrategia en biotecnología debe constituir una referencia para la colaboración entre diferentes agentes (autoridades públicas comunitarias, nacionales y locales, operadores económicos, comunidad científica, etc.). Como parte de la Estrategia de Lisboa, se debe poner en marcha una acción coherente en materia de biotecnología y ciencias de la vida utilizando los métodos existentes de coordinación abierta y evaluación comparativa. Por otro lado, se deben fomentar nuevas formas de colaboración y asociación entre las partes interesadas. Junto con los Estados miembros, la Comisión evaluará si las pautas de competencia y los mecanismos de cooperación actuales permiten alcanzar de forma eficaz los objetivos estratégicos, incluida una reevaluación de si es necesario reforzar las competencias de la Unión Europea de conformidad con el Tratado. A nivel comunitario, estas cuestiones solo se pueden abordar parcialmente ya que la mayoría dependen de otros agentes públicos y privados. Algunos ámbitos como la autorización de productos, el control del mercado interior o las políticas agrícolas o comerciales son competencia exclusiva de la UE. En otros aspectos, la UE, o no tiene competencia, o la

\footnotetext{
${ }^{9}$ Comunicación de la Comisión al Consejo, al Parlamento Europeo, al Comité Económico y Social Europeo y al Comité de las Regiones: "Ciencias de la vida y Biotecnología. Una estrategia para Europa". Bruselas, 23/1/2002 $\operatorname{COM}(2002) 27$ final. Comunicación de la Comisión al Consejo, al Parlamento Europeo, al Comité Económico y Social Europeo y al Comité de las Regiones sobre el "Informe intermedio relativo a la estrategia en el ámbito de las ciencias de la vida y la biotecnología". Bruselas, 10/4/2007 COM(2007) 175 final.
} 
comparte con los Estados miembros. Por lo tanto, en última instancia, la responsabilidad sobre el éxito o el fracaso es una responsabilidad compartida ${ }^{10}$.

Al mismo tiempo, el derecho derivado de la biotecnología, constituido esencial pero no únicamente por la Directiva 2001/18/CE ${ }^{11}$, se asienta sobre la base jurídica de la libre circulación de productos, en este caso OMG, aunque deben protegerse el medio ambiente y la salud humana:

Sin perjuicio de lo dispuesto en el artículo 23, los Estados miembros no podrán prohibir, restringir o impedir la comercialización de OMG que sean productos o componentes de un producto si cumplen las disposiciones de la presente Directiva.

Este es uno de los principales puntos de conflicto de la Directiva, pues, junto con el artículo 23 sobre la cláusula de salvaguarda que se expresa en los siguientes términos, ha sido el caballo de batalla sobre el procedimiento de autorización, o más bien sobre el modelo de autorización existente en la UE en materia de OMG:

Cuando, por disponer de información nueva o adicional con posterioridad a la fecha de la autorización que afecte a la evaluación del riesgo para el medio ambiente o de una nueva valoración de la información existente a tenor de los conocimientos científicos nuevos o adicionales, un Estado miembro tenga razones suficientes para considerar que un OMG que sea un producto o un componente de un producto y que haya sido debidamente notificado y autorizado por escrito de conformidad con la presente Directiva, constituye un riesgo para la salud humana o el medio ambiente, podrá restringir o prohibir provisionalmente en su territorio el uso o la venta de dicho OMG que sea un producto o un componente de un producto.

Los Estados miembros garantizarán que en caso de riesgo grave, se aplicarán medidas de emergencia, tales como la suspensión o el cese de la comercialización, incluida la información al público.

\footnotetext{
${ }^{10}$ Comunicación de la Comisión al Consejo, al Parlamento Europeo, al Comité Económico y Social Europeo y al Comité de las Regiones: "Ciencias de la vida y biotecnología - Una estrategia para Europa". COM/2002/0027 final. DOUE ${ }^{\circ}$ C 055 de 02/03/2002 p. $0003-0032$.

${ }^{11}$ Artículo 22 de la Directiva 2001/18/CE del Parlamento Europeo y del Consejo, de 12 de marzo de 2001, sobre la liberación intencional en el medio ambiente de organismos modificados genéticamente. DOUE de 17 de abril de 2001. L 106/1.
} 
Conjugando ambos preceptos (22 y 23), ha resultado que los últimos consejos de ministros de Medio Ambiente de la UE (marzo y junio de 2014) se han planteado un cambio de legislación sobre los cultivos de transgénicos ante la imposibilidad de llegar a un acuerdo de los 28 países en este punto ${ }^{12}$.

No puede obviarse el trasfondo político y unos antecedentes que deben ponerse de manifiesto en lo que concierne al cumplimiento del sistema legal europeo sobre OMG. En marzo del presente año (2014) se ha reabierto el debate sobre la reforma legislativa de los transgénicos, que ya se propuso en 2010. La Propuesta de Reglamento del Parlamento Europeo y del Consejo por el que se modifica la Directiva 2001/18/CE en lo que respecta a la posibilidad de que los Estados miembros restrinjan o prohíban el cultivo de OMG en su territorio no logra despejar el bloqueo político existente aún ${ }^{13}$. Es esta una discusión interminable que es necesario abordar y cerrar ya que existen nuevas peticiones de autorización sobre otros OMG. Uno de esos productos es el maíz 1507, sobre el cual también se ha pronunciado el TGUE ${ }^{14}$, que declaró que la Comisión había incumplido la Directiva 2001/18/CE al no presentar una propuesta al Consejo de conformidad con al artículo 5.4 de la Decisión comitología 1999/468/CE del Consejo. Tres meses después, el Tribunal se pronunció sobre el caso de la patata Amflora, condenando a la Comisión por incumplimiento.

La propuesta de una mayor subsidiariedad de los Estados miembros en materia de cultivos de OMG se reivindica cada vez más y con mayor intensidad. No obstante, en el Consejo, y pese a los esfuerzos de sucesivas presidencias, sobre todo de la danesa en 2012, no se ha alcanzado un acuerdo por la posición de bloqueo de una minoría de Estados miembros. La Comisión ha proseguido sus esfuerzos para abordar las

\footnotetext{
${ }^{12}$ COUNCIL OF THE EUROPEAN UNION. 3297th Environment Council meeting. Bruselas, 3 de marzo de 2014. $<$ http://www.consilium.europa.eu/ueDocs/cms_Data/docs/pressData/en/envir/141298.pdf $>$

${ }^{13}$ Environment Council meeting. Bruselas, 3 de marzo de 2014. (3297th Council meeting). Proposal for a Regulation of the European Parliament and of the Council amending Directive 2001/18/EC as regards the possibility for the Member States to restrict or prohibit the cultivation of GMOs in their territory. El contenido de este documento no está disponible al público. Información en

$<$ http://register.consilium.europa.eu/content/out?lang=ES\&typ=ENTRY\&i=SMPL\&DOC_ID=ST\%2067 $69 \% 202014 \% 20$ INIT>

${ }^{14}$ Sentencia del Tribunal General de la Unión Europea de 26 de septiembre de 2013. Asunto T-164/10, Pioneer Hi-Bred International, Inc., contra la Comisión Europea. Judgment of the General Court, 26 September 2013. In Case T-164/10, Pioneer Hi-Bred International, Inc., versus European Commission.
} 
preocupaciones de estos Estados miembros en su posición de bloqueo, al tiempo que obtiene el apoyo de la gran mayoría de los Estados miembros a la propuesta.

Recientemente, el Consejo, en su reunión y formación de ministros de Medio Ambiente, alcanzó un acuerdo para proponer - como respuesta a la petición dirigida a la Comisión por trece Estados miembros en junio de 2009- una sólida base jurídica en el marco legal de la UE con el fin de permitir que los Estados miembros puedan restringir o prohibir los cultivos de OMG en su territorio pese a que hayan sido autorizados en la $\mathrm{UE}^{15}$

Los eurodiputados votaron el 13 de enero de 2015 a favor de que los Estados puedan restringir o prohibir cultivos de OGM en sus respectivos territorios incluso aunque hayan sido aprobados por el conjunto de la Unión Europea. La futura normativa (modificación de la Directiva 2001/18/CE debe aún pasar por el Consejo de la UE para que los Estados hagan una segunda lectura. Si el Consejo la adopta, la nueva directiva entrará en vigor con efecto inmediato.

\section{Medio ambiente y cambio climático}

El artículo 191 del TFUE hace de la lucha contra el cambio climático un objetivo expreso de la política medioambiental de la UE:

1. La política de la Unión en el ámbito del medio ambiente contribuirá a alcanzar los siguientes objetivos:

la conservación, la protección y la mejora de la calidad del medio ambiente,

la protección de la salud de las personas,

la utilización prudente y racional de los recursos naturales,

el fomento de medidas a escala internacional destinadas a hacer frente a los problemas regionales o mundiales del medio ambiente y en particular a luchar contra el cambio climático.

2. La política de la Unión en el ámbito del medio ambiente tendrá como objetivo alcanzar un nivel de protección elevado, teniendo presente la diversidad de situaciones existentes en las distintas regiones de la Unión. Se basará en los

\footnotetext{
${ }^{15}$ Un resumen de lo acontecido en el último Consejo de ministros de Medio Ambiente de la UE el pasado 12 de junio de 2014 puede consultarse aquí:

$<$ http://www.consilium.europa.eu/uedocs/cms_data/docs/pressdata/en/envir/143178.pdf>.
} 
principios de cautela y de acción preventiva, en el principio de corrección de los atentados al medio ambiente, preferentemente en la fuente misma, y en el principio de quien contamina paga.

En este contexto, las medidas de armonización necesarias para responder a exigencias de la protección del medio ambiente incluirán, en los casos apropiados, una cláusula de salvaguardia que autorice a los Estados miembros a adoptar, por motivos medioambientales no económicos, medidas provisionales sometidas a un procedimiento de control de la Unión.

3. En la elaboración de su política en el área del medio ambiente, la Unión tendrá en cuenta:

los datos científicos y técnicos disponibles, las condiciones del medio ambiente en las diversas regiones de la Unión, las ventajas y las cargas que puedan resultar de la acción o de la falta de acción, el desarrollo económico y social de la Unión en su conjunto y el desarrollo equilibrado de sus regiones.

$[\ldots]$

Cabe destacar el objetivo de la utilización racional y prudente de los recursos naturales y otros puntos de este precepto, pero, como en todos los apartados de este estudio, los campos de interés son muchos y desbordarían el objeto de esta aportación a la RCDA. Sin embargo, ateniéndonos a lo que ahora interesa, es preciso destacar que el impacto sobre el medio ambiente agrícola ocasionado por el cambio climático viene determinado por las nuevas condiciones (temperaturas invernales más suaves) y un cambio potencial de las especies/variedades cultivadas que podrían favorecer el brote de plagas y enfermedades y alterar su distribución. Asimismo, algunas de las "malas hierbas" exóticas de los cultivos podrían resultar favorecidas bajo las nuevas condiciones. Como en el caso del sector forestal, podría producirse un aumento en el uso de pesticidas y agentes de control biológico exóticos, con consecuencias potencialmente negativas para la biodiversidad, así como un incremento en la implantación de cultivos modificados genéticamente (por ejemplo, resistentes a los herbicidas), que podrían actuar como contaminantes de la biodiversidad autóctona ${ }^{16}$.

\footnotetext{
${ }^{16}$ CAPDEVILA-ARGÜELLES, L., ZILLETTI, B. y SUÁREZ ÁlVAREZ, V.A., Cambio climático y especies exóticas invasoras en España. Diagnóstico preliminar y bases de conocimiento sobre impacto y vulnerabilidad, Oficina Española de Cambio Climático, Ministerio de Medio Ambiente, y Medio Rural y
} 
Lo más destacable de la protección ambiental es que es un deber constitucional y una función pública transversal. La trascendencia material de sus postulados y la extensión cada vez mayor de su radio de acción han transformado el ordenamiento ambiental de un conjunto disperso de normas sectoriales a un sistema cada vez más homogéneo de principios y preceptos presentes de forma horizontal en muchas de las políticas verticales a nivel comunitario y estatal. Como ha señalado la propia Unión Europea, las consideraciones ecológicas deben tenerse en cuenta a la hora de formular y aplicar políticas económicas y sectoriales, en las decisiones de los poderes públicos, en la dirección y el desarrollo de los procesos de producción y en el comportamiento y las elecciones personales ${ }^{17}$.

No obstante, en esta relación medio ambiente y cambio climático existen investigaciones en relación con la integración de estas dos políticas en las demás. Se cuestiona si la integración de la política del clima es "más fuerte" que otros tipos de integración de política ambiental, existiendo razones para pensar que la integración de la política del clima puede llegar a ser, en igualdad de otras condiciones, más fuerte o más exitosa que la integración de la política ambiental en general o que la integración de otros aspectos de la política ambiental. Por ejemplo, se argumenta que en la UE el proceso de Cardiff perdió rápidamente mucho de su ímpetu, mientras que los programas europeos de cambio climático continúan y se van renovando ${ }^{18}$.

\section{Energía y biocarburantes}

La evolución normativa puede resumirse siguiendo a MELLADO RUIZ: “A pesar de que $[\ldots]$ el sector de la energía ha sido, tradicionalmente, uno de los campos con mayores limitaciones de intervención por parte de la UE, en virtud de la operatividad de los principios de subsidiariedad y soberanía permanente sobre los recursos energéticos

Marino, Madrid, 2011, 146 pp. Accesible en <http:/www.magrama.gob.es/es/ceneam/grupos-de-trabajoy-seminarios/red-parques-

nacionales/Cambio_climático_y_especies_exóticas_Invasoras_en_españa_tcm7-326551.pdf> Página 125.

${ }^{17}$ Páginas 13 y 14 del artículo publicado en Actualidad Jurídica Ambiental por MELLADO RUIZ, L., "Bases teóricas y manifestaciones jurídico-sectoriales de los nuevos enfoques de sostenibilidad agrícola"; <http://www.actualidadjuridicaambiental.com/wp-content/uploads/2012/01/JUNIO-20121.pdf>

${ }^{18}$ Véase la interesante aportación en este sentido de la investigación de HANF, K., MEZO, J., LANAIA, A. y MORATA, F., "Integración ambiental de las políticas en el sistema autonómico español: un reto aún no superado", ponencia para el XI Congreso de la Asociación Española de Ciencia Política, Sevilla, septiembre de 2013, pp. 14 y 15 . Accesible en

$<$ http://www.aecpa.es/uploads/files/modules/congress/11/papers/1005.pdf $>$ 
naturales, lo cierto es que, en las últimas décadas, las competencias comunitarias, al menos en sede de armonización o aproximación regulatorias, no han dejado de incrementarse, así como por la interacción de otros títulos competenciales vinculados (singularmente, la protección ambiental, el aseguramiento de un mercado único, la PAC, etc.). Del desinterés comunitario por la energía se ha pasado, en un proceso lógico, a su entronización basilar como pilar fundamental del desarrollo económico, y como eje directriz de la materialización de las políticas medioambientales. Esto se ha traducido también, en el campo más específico de las energías renovables, en una creciente preocupación comunitaria $\mathrm{y}$, consiguientemente, en una sucesión de propuestas y recomendaciones, seguidas de normas programáticas o indicativas $\mathrm{y}$, finalmente, de auténticas normas vinculantes, aún con diferente grado de imperatividad en cuanto a sus contenidos, para los Estados miembros ${ }^{19}$.

Después de este resumen, se trazan ahora las últimas líneas de acción europea sobre energía, donde se afirma que un mercado interior de la electricidad para Europa no es un fin en sí mismo, sino que se trata de algo urgentemente necesario para lograr los objetivos de la política energética de la Unión (art. 194 TFUE). Así lo determinó la Comisión Europea en noviembre de $2013^{20}$ en su Comunicación sobre el diseño de esquemas de apoyo a las renovables y la intervención pública, donde apunta hacia la necesidad de que las plantas de producción de energía eléctrica a partir de fuentes renovables vayan percibiendo remuneraciones ligadas al mercado en lugar de regímenes retributivos basados en instrumentos de carácter administrativo, opinando que los mecanismos de intervención pública para el desarrollo de las energías renovables que existen en los distintos Estados miembros muestran deficiencias en cuanto a los esquemas de apoyo para estas tecnologías.

Así, el objetivo fijado para las energías renovables a escala europea se alcanzaría mediante un nuevo marco de gobernanza basado en planes nacionales para una energía competitiva, segura y sostenible elaborados por los Estados miembros. Algunos de ellos ya han establecido objetivos ambiciosos en materia de fuentes de energía renovables

\footnotetext{
${ }^{19}$ MELLADO RUIZ, L., "Obligación de uso de biocarburantes y biogás”, Mellado Ruiz, L. (coord.), Energías renovables, ahorro y eficiencia energética en Andalucía. Régimen jurídico, Atelier, 2012, pp. 98 у 99.

${ }^{20}$ COMUNICACIÓN DE LA COMISIÓN, "Realizar el mercado interior de la electricidad y sacar el máximo partido de la intervención pública", Bruselas, 5/11/2013 C(2013) 7243 final. Accesible en <http://ec.europa.eu/energy/gas_electricity/doc/com_2013_public_intervention_es.pdf>
} 
para 2030 y años posteriores que conllevarán progresos sustanciales en pos del objetivo de la UE. Cada Estado miembro debe dejar claro su compromiso con las energías renovables indicando cómo se alcanzaría ese objetivo y teniendo en cuenta la necesidad de cumplir la normativa de competencia y ayudas estatales a fin de evitar el falseamiento del mercado y garantizar una relación coste-eficacia aceptable ${ }^{21}$.

Entre los esfuerzos realizados en la UE para la lucha contra el cambio climático, y por lo que respecta al presente estudio y los biocombustibles, la normativa en vigor que representa la Directiva sobre energías renovables (2009/28/CE) se propone garantizar que, para 2020, energías renovables como la biomasa y las energías hidráulica, eólica y solar representen al menos el $20 \%$ del consumo total de energía de la UE en términos de generación de electricidad, transporte, calefacción y refrigeración. Dentro del objetivo general se estableció un objetivo mínimo vinculante para cada Estado miembro que era el de conseguir que al menos un $10 \%$ de la energía consumida para el transporte fuese renovable. Sin embargo, este objetivo tendrá carácter vinculante "siempre y cuando la producción sea sostenible" y "los biocarburantes de segunda generación estén disponibles comercialmente".

En el contexto de la revisión de la Directiva sobre energías renovables, el Parlamento se ha esforzado por lograr que la nueva legislación incluyese criterios para garantizar que la producción de biocarburantes se ajustase a la sostenibilidad social y medioambiental y no condujese a la deforestación ni al aumento de los precios de los alimentos. El Parlamento instó a que se impusiese un límite a los biocarburantes de primera generación procedentes de fuentes tradicionales derivadas de alimentos, que estableció en el 6\% del consumo final de energía en el sector del transporte para 2020 (frente al límite del 10\% que está actualmente en vigor), y también a que se adoptasen rápidamente los carburantes avanzados fabricados a partir de algas o determinados tipos de residuos.

En el ámbito interno, ese ajuste con la sostenibilidad social y ambiental en la producción de biocarburantes se encuentra regulada ${ }^{22}$, determinado que el director

\footnotetext{
${ }^{21}$ Comunicación de la Comisión sobre clima y energía hasta 2013: COMUNICACIÓN DE LA COMISIÓN AL PARLAMENTO EUROPEO, AL CONSEJO, AL COMITÉ ECONÓMICO Y SOCIAL EUROPEO Y AL COMITÉ DE LAS REGIONES, "Un marco estratégico en materia de clima y energía para el período 2020-2030", Bruselas, 22/1/2014 COM(2014) 15 final, pp. 7 y 8.

${ }^{22}$ Real Decreto 1597/2011, de 4 de noviembre, por el que se regulan los criterios de sostenibilidad de los biocarburantes y biolíquidos, el Sistema Nacional de Verificación de la Sostenibilidad y el doble valor de algunos biocarburantes a efectos de su cómputo. BOE de 5 de noviembre de 2011.
} 
general de Política Energética y Minas dictará, mediante resolución, las disposiciones necesarias para definir con detalle los elementos del sistema de verificación de la sostenibilidad necesarios para su puesta en funcionamiento, que podrán incluir, entre otras, la información sobre los aspectos sociales y medioambientales suplementarios que deben enviar los agentes, según lo establecido en la letra d) del apartado 1 del artículo $11^{23}$.

Por último, y en lo que respecta a España, en los informes de supervisión de la CNE para los biocarburantes de los años 2009 y 2010 (únicos publicados encontrados ${ }^{24}$ ) se echa en falta una mayor información acerca del tipo de materias primas utilizadas y el origen en la elaboración de los biocarburantes. Se habla del empleo de aceite de palma, colza, soja, etc., pero no del origen de estas materias. Ciertamente, puede ser difícil asignarle esta tarea al órgano regulador integrado ahora en la Comisión Nacional de los mercados y la competencia, pero sí es exigible una mayor coordinación con otros organismos públicos y departamentos ministeriales que deben tener la información y facilitarla $^{25}$. Bien es verdad que el contexto de cambio ocasionado por la creación de la nueva CNMC ha hecho más difícil encontrar este tipo de información por la reordenación de funciones y actividades que realiza el nuevo órgano. La recientemente creada CNMC asume bastantes funciones de supervisión y control de los mercados (de comunicaciones electrónicas, eléctrico y del gas natural, del mercado postal, de las comunicaciones audiovisuales y ferroviario) propias de los organismos reguladores que absorbe; sin embargo, una de las principales novedades de la Ley de creación de la CNMC es la recuperación de diversas funciones por parte de varios ministerios. En concreto, el Ministerio de Industria, Energía y Turismo recupera, en el sector de la

\footnotetext{
${ }^{23}$ Disposición final tercera (apartado 5) relativa a las facultades de desarrollo y aplicación del Real Decreto 1597/2011, de 4 de noviembre.

$24<$ http://www.cne.es/cgi-bin/BRSCGI.EXE?CMD=VERDOC\&BASE=PUBL\&docr=3\&SORT=FECH $\&$ RNG $=20 \&$ SEPARADOR $=\&$ CODI-C $=$ ANUALES $\% 20$ NO $\% 20 I N G L E S \& T I T U-$ $\mathrm{C}=\mathrm{BIOCARBURANTES \& op}=\mathrm{Y} \& \mathrm{op}=$ undefined $\& \mathrm{op}=$ undefined\&op $=$ undefined\&op $=\mathrm{Y} \& \mathrm{op}=\mathrm{Y} \&>$ INFORME PARA EL EJERCICIO 2009. < 2 http://www.cne.es/cgibin/BRSCGI.EXE?CMD $=$ VERDOC $\&$ BASE $=$ PUBL $\&$ docr $=2 \&$ SORT $=-$ FECH\&RNG $=20 \&$ SEPARADOR $=\&$ CODI-C $=$ ANUALES $\% 20$ NO $\% 20$ INGLES\&TITU$\mathrm{C}=\mathrm{BIOCARBURANTES \& op}=\mathrm{Y} \& \mathrm{op}=$ undefined $\& \mathrm{op}=$ undefined\&op $=$ undefined\&op $=\mathrm{Y} \& \mathrm{op}=\mathrm{Y} \&>$ INFORME PARA EL EJERCICIO 2010.

${ }^{25}$ Véase gráfico de la página 35 del Informe de supervisión del 2010: Gráfico 3.2.3. Tipos y orígenes de las materias primas de biodiésel consumido en España en 2010 (datos en $\mathrm{m}^{3}$ ).
} 
energía y específicamente en el de los hidrocarburos, la expedición de los certificados y la gestión del mecanismo de certificación de consumo y venta de biocarburantes ${ }^{26}$.

Volviendo a los resultados encontrados para los biocarburantes utilizados a nivel interno, el biodiésel consumido en España se caracteriza por lo siguiente ${ }^{27}$ :

1. Ha sido producido principalmente a partir de soja argentina (34\%) y palma indonesia (30,55\%). Argentina ha superado en 2010 a Indonesia como principal país de origen de la materia prima (19,21\% en 2009 frente a 34,02\% en 2010), aunque Indonesia ha incrementado también su participación (del 25,12\% al 30,61\%), lo que ha implicado una concentración de los orígenes en torno a estos dos países (65\% en 2010 frente a 43,88\% en 2009) a costa de la reducción de la participación de la soja brasileña (7,42\% en 2010 frente a $16,19 \%$ en 2009$)$.

2. En total, la soja y la palma han supuesto más del $85 \%$ de las materias primas empleadas en su fabricación $(86,02 \%)$.

3. España ha suministrado el $8,29 \%$ de las materias primas empleadas para la fabricación del biodiésel consumido en 2010 (12,49\% en 2009).

4. El biodiésel se ha fabricado principalmente en España (54,56\%), aunque en menor proporción que en 2009 (69,12\%). El segundo país con mayor participación ha sido Argentina (27,23\%), que ha duplicado su peso respecto al año anterior.

Hay que tener en cuenta, además, que las regulaciones sobre cultivos modificados genéticamente en Argentina o Brasil pueden llegar a ser incompatibles con la legislación europea. En este sentido, se puede afirmar que el principio de precaución no es usado en países fuera del ámbito de la UE, pero sí otros como el de equivalencia sustancial más laxos en la comercialización de OMG.

\section{Política Agrícola Común (PAC) e industria agroalimentaria}

Fuera del alcance de este trabajo se sitúa el completo análisis de los siguientes dos aspectos: la política agrícola y la alimentación, y, más aún, todas las interacciones

\footnotetext{
${ }^{26}$ NAVARRO RODRÍGUEZ, P., "La nueva Comisión Nacional de los Mercados y la Competencia: naturaleza, régimen jurídico e independencia”, Revista General de Derecho Administrativo, núm. 34, Iustel, 2013, pp. 1-52 y p. 23.

${ }^{27}$ Página 36 del Informe anual sobre el uso de biocarburantes correspondiente al ejercicio 2010. Publicado el 19 de enero de 2012.
} 
existentes entre estos campos y el cambio climático y la protección del medio ambiente. Solamente tres aportaciones a tener en cuenta. En primer lugar, la visión que de la nueva PAC se tiene en España. Dentro de las tendencias normativas en el sector agroalimentario, después de haber sido históricamente protegido, parece que la apertura de mercados es ahora innegociable por la globalidad de mercados (PAC 2014-2020). La apertura a nuevos mercados conllevará que cada vez más las empresas se vean sometidas a más normativas y convenios e incidirá directamente en el potencial competitivo de las empresas ${ }^{28}$.

En segundo lugar, cabe decir que, en el marco de los objetivos y principios establecidos a nivel comunitario, la consecución de un "desarrollo agrario sostenible" a nivel interno impone cambios importantes en los sistemas de ordenación de las actividades agropecuarias. El carácter multifuncional de la agricultura y sus evidentes interacciones con los postulados del principio de sostenibilidad imponen una nueva regulación integrada superadora de los enfoques sectoriales y parciales existentes hasta ahora ${ }^{29}$.

En tercer lugar, hay que tener en cuenta también que existe especulación en los precios de los alimentos propiciada por los biocombustibles. Pero, aunque se controlara de nuevo el precio de los alimentos, aún quedan entre 800 y 1.000 millones de personas que pasan hambre por permanecer atrapadas en la agricultura tradicional de subsistencia. La revolución verde dio todo lo que podía dar de sí, por lo que ahora se está a las puertas de lo que se llama revolución genética ${ }^{30}$. No obstante, frente a esta revolución genética no puede dejar de señalarse la necesidad de regular de manera clara y segura la convivencia o coexistencia de la agricultura convencional, la ecológica y la modificada genéticamente, pues son las permitidas por la legislación y hacer compatible su convivencia no ha sido tarea fácil hasta el momento. Y creemos que no lo será en el futuro ya que la evolución y el peso de cada uno de estos modelos dependerán de la

\footnotetext{
${ }^{28}$ MARCO ESTRATÉGICO PARA LA INDUSTRIA DE ALIMENTACIÓN Y BEBIDAS, Ministerio de Agricultura, Alimentación y Medio Ambiente y FIAB (Federación de Industrias de Alimentación y Bebidas), 2014.

${ }^{29}$ MELLADO RUIZ, L., "Bases teóricas y manifestaciones jurídico-sectoriales de los nuevos enfoques de sostenibilidad agrícola", Actualidad Jurídica Ambiental, ISSN-e 1989-5666, núm. 3 (junio), 2012, pp. 120. El artículo analiza las bases teóricas de dicha imbricación y sistematiza las fórmulas normativas actuales tanto de condicionalización ambiental directa de la agricultura como de aplicación del principio de sostenibilidad económica a las actividades de producción agrícola. Accesible en

http://www.actualidadjuridicaambiental.com/wp-content/uploads/2012/05/Bases-Teóricas-yMenifestaciones-Jur\%C3\%ADdico-Sectoriales-de-los-Nuevos-Enfoques-de-SostenibilidadAgr\%C3\%ADcola.pdf

${ }^{30}$ LOZANO LEYVA, M., El fin de la ciencia, Ed. DEBOLS!LLO, 2013, p. 378.
} 
evolución de la demanda internacional. El mantenimiento de las tendencias recientes, especialmente el cambio de la demanda alimentaria en Asia y en los países emergentes, implicará la necesidad de crecimiento del modelo convencional ${ }^{31}$.

Por último, el clima también es una variable que determina el tipo de cultivo posible en un área determinada. En relación con los biocarburantes, los cultivos tropicales de caña de azúcar permiten una producción media por hectárea de 6.000 litros de bioetanol en Brasil, mientras que en Europa, al emplearse cereales, se limita este rendimiento a menos de la mitad. Asimismo, en Europa se produce gran cantidad de biodiésel procedente de aceite de colza, principalmente en Alemania y Francia ${ }^{32}$.

\section{A MODO DE CONCLUSIÓN}

Existe una paradoja que nos enfrenta a un problema complejo que es global y que se afronta con una nueva tecnología que, a su vez, puede provocar otra complicación integral. Ante este riesgo continuo de crisis sucesivas, debe imponerse - como primera medida - la precaución máxima. En la explicación de esta incongruencia hay que mencionar la existencia de un optimismo que gravita alrededor de dos fuerzas o potencias. La primera potencia ${ }^{33}$ se encuentra en la necesidad del progreso y del

${ }^{31}$ GARRIDO COLMENERO, A., ATANCE MUÑOZ, I. y GÓMEZ RAMOS, A., "Agricultura, Alimentación, Biocarburantes y Medio Ambiente", Revista Economía y Medio Ambiente, marzo-abril de 2009 , núm. 847 , ICE, pp. 41-57 y p. 56. En relación con la crisis alimentaria y el derecho a la alimentación, véase el libro Fed up with the right to food? The Netherlands' policies and practices regarding the human right to adequate food, que edita la cátedra de Derecho Alimentario de la Universidad de Wageningen, dirigida por el profesor B. van der Meulen. En el capítulo 8, página 121, O. HOSPES afirma: "Es sobradamente conocido que el incremento del uso de alimentos como el maíz para producir energía ha sido causa de la crisis global alimentaria acontecida en 2007-2008, sumándose a esto las severas sequías, la escasez, los altos precios de los combustibles fósiles, la creciente demanda de alimentos, la falta de inversión en la agricultura y la intensificación de las restricciones a la exportación". El autor reafirma esto con el apoyo de informes de la OCDE, la FAO y el Banco Mundial. OCDE, Is the cure worse than disease? Report of the Round Table on Sustainable Deveploment of the OECD, 2007; OCDE y FAO, OECD-FAO Agricultural Outlook 2007-2016, 2007; BANCO MUNDIAL, Rising food prices: policy options and World Bank response, 2007.

${ }^{32}$ Evaluación Preliminar de los Impactos en España por Efecto del Cambio Climático, 2005. (Impactos sobre el sector energético, p. 630). Última consulta el 12 de octubre de 2014 $<$ http://www.magrama.gob.es/es/cambio-climatico/temas/impactos-vulnerabilidad-yadaptacion/evaluacion_preliminar_impactos_completo_2_tcm7-12439.pdf $>$

${ }^{33}$ Eurobarómetro de 1991, OPINIONS OF EUROPEANS ON BIOTECHNOLOGY IN 1991. E1 Eurobarómetro llevado a cabo en la totalidad de la Comunidad Europea (por aquel entonces eran solo doce países los miembros de la Comunidad, actual Unión Europea) registró un dato curioso para nuestro país cual fue el de situarlo en el primer lugar del ranking sobre "optimismo global" de las nuevas tecnologías y sus efectos anticipados, con especial hincapié en las siete nuevas tecnologías propuestas: energía solar, información tecnológica, biotecnología, ingeniería genética, telecomunicaciones, nuevos materiales y sustancias y exploración espacial. Sin embargo, ha habido un cambio significativo de 
desarrollo económico que quiebra con la crisis ecológica y la económica: la crisis integral del sistema. Pero esta nueva dificultad se solventa con más desarrollo de nuevas tecnologías y de un crecimiento y avance de la ciencia y de la investigación imparables, es el optimismo de segunda potencia.

Explicando de otro modo dicha contradicción, puede decirse que, ante problemas globales como el cambio climático, el hambre en el mundo o la escasez energética en sus fuentes tradicionales, junto con el impacto ambiental que todo ello conlleva, la solución se halla en la búsqueda de nuevas fórmulas energéticas como los biocarburantes o, en sentido amplio, la bioenergía:

[...] se reclama una sexta ola basada en tecnologías inteligentes que nos lleve hasta 2050. "Necesitamos una sexta ola de tecnologías sostenibles y ecológicas [...] para poder asegurar la subsistencia de todos los habitantes del planeta de una forma que resulte sostenible en el plano medioambiental ${ }^{34,}$.

El ejemplo tecnológico que contribuye a la solución del hambre a través de la biotecnología comporta, al mismo tiempo, importantes riesgos de seguridad ambiental y consumo de recursos naturales. $\mathrm{Y}$ el problema se agrava cuando se contemplan estas dos soluciones tecnológicas en interacción. Los biocarburantes desplazan y alteran la producción alimentaria, y la biotecnología requiere condiciones especiales que implican más recursos económicos y naturales que conducen finalmente a que la introducción de esas tecnologías no resuelve, atenúa o mitiga el riesgo en modo alguno, sino que lo incrementa al exigirse correcciones o modulaciones.

La evaluación ambiental del cambio de combustibles como innovación tecnológica debe atender no solo a los efectos locales, sino también a los globales, sociales y económicos. En el caso de los agrocombustibles, para alcanzar el objetivo de la UE de reemplazar el $5,75 \%$ de los combustibles fósiles con biocarburantes es necesario dedicar a cultivos

opinión de la ciudadanía europea ante la biotecnología aplicada a la alimentación, tal como señalan ESPEITX BERNAT, E., CANTARERO ABAD, L., MEDINA, F. X. y CÁCERES NEVOT, J., "El papel de la información en las cogniciones y percepciones hacia nuevas tecnologías aplicadas a los alimentos", Revista Política y Sociedad, 51, núm. 1, 2014, pp. 95-120, accesible en: http://dx.doi.org/10.5209/rev_POSO.2014.v51.n1.42482. Así, en la página 97, indican: "Según los datos recogidos en el conjunto de países de la Unión Europea, el rechazo alcanzaría a tres cuartas partes de la población. Lo mismo sucede con la utilización de la clonación animal para la obtención de productos alimentarios, que genera la oposición de más del $80 \%$ de la población".

${ }^{34}$ JEFFREY SACHS, "Precisamos una sexta ola de tecnologías sostenibles y ecológicas", 12 de junio de 2014, edición 2014 de la Semana Verde en Bruselas. Jeffrey Sachs es economista y asesor experto de Naciones Unidas. Accesible en <http://cordis.europa.eu/news/rcn/36601_es.html>. 
energéticos alrededor del $20 \%$ del suelo cultivable de la UE, lo que significa que hacen falta importaciones de materias primas procedentes de otros países, con los consiguientes interrogantes sociales. Además, se han señalado algunas dudas sobre las consecuencias globales: "Los ahorros en términos de energía fósil y gases de efecto invernadero derivados del uso de los biocombustibles como el etanol o el biodiesel producidos de modo convencional dependen estrechamente del proceso de fabricación y del destino de los subproductos residuales. El balance de emisiones de gases de efecto invernadero es particularmente incierto a causa de las emisiones de óxidos de nitrógeno de la agricultura ${ }^{35 \%}$.

Este planteamiento nos somete a una espiral de sofisticación de los problemas que desconocemos si es sostenible o no. El cambio de orientación legislativa en las energías renovables sería una aplicación implícita del principio de precaución como principio general del derecho comunitario. La vigente Directiva sobre energías renovables no lo menciona explícitamente, pero de su lectura se traduce una cierta cautela.

Sin perder de vista los problemas de la subsidiariedad frente a la acción comunitaria europea integral, si se está ante un cambio global, ¿existe un regulador que abarque toda esa globalidad? ¿Dónde queda la subsidiariedad entonces? Se ha dicho, y con razón, que el ejemplo de la crisis alimentaria no es solo un asunto nacional o de nuevas tecnologías, sino que es un ejemplo dramático del fracaso de la gobernanza y la complejidad de una nueva era global. Muchos actores estatales y no estatales están de acuerdo en las complejas causas de los dramáticos aumentos de precios de los alimentos en los años 2007-2008, pero al mismo tiempo casi no consideran las implicaciones del fracaso de los Estados para hacer frente a esta crisis - ya sea individual o colectivamente como miembros de instituciones y organismos internacionales- Los gobiernos solamente definen cuestiones clave y proponen principios generales $\mathrm{o}$ soluciones técnicas para hacer frente a la crisis alimentaria (como una mayor inversión en la agricultura y en tecnologías como los biocombustibles de segunda generación), pero son escasas las voces que claman por una redefinición de las responsabilidades de

\footnotetext{
35 "La estrategia española de movilidad sostenible y los gobiernos locales", 2010, p. 115. Este documento apoya la aplicación en el ámbito municipal de la Estrategia Española de Movilidad Sostenible, aprobada en abril de 2009.
} 
los actores estatales y no estatales, nuevos arreglos institucionales y nuevas formas de gobierno al mismo tiempo ${ }^{36}$.

Ante el progreso imparable de la ciencia, la investigación y las tecnologías que se derivan de los grandes avances, se ha afirmado que existe una nueva relación entre Estado y sociedad, y, desde la perspectiva interna estatal, se propugna por la doctrina española lo siguiente: “[...] el Estado, careciendo ya de la titularidad sobre los medios instrumentales, tecnológicos y humanos, para desarrollar o dirigir la investigación científica, habrá de garantizar —o pretenderlo al menos- que los progresos de la investigación y de la ciencia sean en beneficio del interés general, tal como afirma la propia Constitución 37 ". Asimismo, se señala: "Se ha producido un vuelco que ha situado ese conocimiento y la investigación científica mayoritaria y decisivamente en la órbita privada".

En definitiva, y desde el enfoque de las políticas sectoriales europeas mencionadas al principio de este artículo, se reivindica una mayor eficacia y coherencia. Así: “[...] las medidas que deben ser eficaces y oportunas deben producir los resultados buscados sobre la base de unos objetivos claros. La eficacia requiere también una aplicación proporcionada de las políticas y que las decisiones se apliquen en el nivel más apropiado. En cuanto a la coherencia, las políticas desarrolladas y las acciones emprendidas deben ser coherentes y fácilmente comprensibles. La necesidad de coherencia de la Unión es cada vez mayor, pues sus tareas son cada vez más complejas, [...]; los desafíos tales como el del cambio climático o la evolución demográfica, rebasan las fronteras de las políticas sectoriales que han cimentado la construcción de la Unión; y las autoridades regionales y locales están cada vez más implicadas en las políticas comunitarias. La coherencia requiere, por tanto, un liderazgo político y un firme compromiso por parte de las Instituciones con vistas a garantizar un enfoque coherente dentro de un sistema complejo ${ }^{38 \%}$.

\footnotetext{
${ }^{36}$ HOSPES, OTTO, "Regulating biofuels in the name of sustainability or the right to food? The case of an emergin policy field in the Netherlands", Hospes, O. y Van der Meulen, B. (eds.), Fed up with the right to food? The Netherlands' policies and practices regarding the human right to adequate food, Wageningen Academic Publishers, The Netherlands, 2009, pp. 121-135 y p. 121.

${ }^{37}$ ESTEVE PARDO, J., La nueva relación entre Estado y sociedad. Aproximación al trasfondo de la crisis, Marcial Pons, 2013, pp. 157, 158 y 159.

${ }^{38}$ PAREJO NAVAJAS, T., La estrategia territorial europea. La percepción comunitaria del uso del territorio, Marcial Pons, 2004, pp. 417 y 418.
} 
El vector principal del "trío ganador" mencionado en la introducción debe ser entonces la lucha contra el cambio climático, pues la adaptación a este es un área de actividad con un marcado carácter transversal que implica a múltiples sectores socioeconómicos y sistemas ecológicos y a múltiples niveles administrativos. La compleja arquitectura institucional y distribución competencial en los niveles europeo, nacional y autonómico $^{39}$ obliga a realizar un elevado esfuerzo de coordinación entre todos los actores para que las acciones de adaptación sean coherentes y eficaces ${ }^{40}$.

\section{BIBLIOGRAFÍA}

CAPDEVILA-ARGÜELLES, L., ZILLETTI, B. y SUÁREZ ÁLVAREZ, V.A., Cambio climático y especies exóticas invasoras en España. Diagnóstico preliminar y bases de conocimiento sobre impacto y vulnerabilidad, Oficina Española de Cambio Climático, Ministerio de Medio Ambiente, y Medio Rural y Marino, Madrid, 2011, pp. 146. Accesible en $\quad<$ http://www.magrama.gob.es/es/ceneam/grupos-de-trabajo-yseminarios/red-parquesnacionales/Cambio_climático_y_especies_exóticas_Invasoras_en_españa_tcm7326551.pdf>.

ELVIRA, A., "El derecho a la investigación científica en el marco de la Unión Europea”, Chueca, R. (dir.), La investigación cientifica como derecho fundamental, Comares, 2012, pp. 69-100.

ESPEITX BERNAT, E., CANTARERO ABAD, L., MEDINA, F. Xavier y CÁCERES NEVOT, J., "El papel de la información en las cogniciones y percepciones hacia nuevas

\footnotetext{
${ }^{39}$ No puede dejar de mencionarse la dificultad ad intra en cada país de esa actuación plural o compuesta. Para un estudio en profundidad, puede verse PÉREZ GABALDÓN, M., La gestión intergubernamental de la política de cambio climático en España, Aranzadi, 2013, p. 361: "La imprecisión en la determinación de los ámbitos competenciales lleva, inevitablemente, a la aparición de zonas en las que se produce una duplicidad de actuaciones y un solapamiento de políticas públicas, lo que revierte negativamente en la consecución del supuesto objetivo común. Esta realidad reposa tanto en la indefinición legal como en el talante de la actuación simultánea del Estado y las Comunidades Autónomas que, en muchos casos, se da sin que los entes competentes atiendan a la presencia de otros poderes públicos actuando sobre la misma realidad y para el mismo fin -a través incluso del mismo instrumento-, así como también para fines que chocan con el propiamente ambiental de la lucha contra el cambio climático".

${ }^{40}$ Siendo la Oficina Española de Cambio Climático la unidad responsable de la coordinación, la gestión y el seguimiento de la implementación del Plan Nacional de Adaptación al Cambio Climático. Véase el propio Plan Nacional de Adaptación al Cambio Climático. III Informe de seguimiento (2014), p. 27. Última consulta el 12 de octubre de 2014: < http://www.magrama.gob.es/es/cambioclimatico/temas/impactos-vulnerabilidad-y-adaptacion/3_informe_seguimiento_pnacc_tcm7-312797.pdf $>$
} 
tecnologías aplicadas a los alimentos”, Revista Política y Sociedad, 51, núm. 1, 2014, 95-120. <http://dx.doi.org/10.5209/rev_POSO.2014.v51.n1.42482>.

ESTEVE PARDO, J., La nueva relación entre Estado y sociedad. Aproximación al trasfondo de la crisis, Marcial Pons, 2013.

GARRIDO COLMENERO, A., ATANCE MUÑOZ, I. y GÓMEZ RAMOS, A., "Agricultura, Alimentación, Biocarburantes y Medio Ambiente", Revista Economía y Medio Ambiente, marzo-abril 2009, núm. 847, ICE, pp. 41-57.

GONZÁLEZ MERINO, A. y CASTANEDA ZAVALA, Y., "Biocombustibles, biotecnología y alimentos: Impactos sociales para México". Argumentos (Méx.) vol. 21, núm. 57, 2008, pp. 55-83.

$<$ http://www.scielo.org.mx/scielo.php?script=sci_arttext\&pid=S0187$57952008000200004 \& \operatorname{lng}=\mathrm{es} \& \mathrm{nrm}=$ iso $>$.

HANF, K., MEZO, J., LANAIA, A. y MORATA, F., "Integración ambiental de las políticas en el sistema autonómico español: un reto aún no superado”, ponencia para el XI Congreso de la Asociación Española de Ciencia Política, Sevilla, septiembre de 2013. Accesible en:

$<$ http://www.aecpa.es/uploads/files/modules/congress/11/papers/1005.pdf.>

HOSPES, O. y VAN DER MEULEN, B., Fed up with the right to food? The Netherlands' policies and practices regarding the human right to adequate food. Wageningen Academic Publishers, The Netherlands, 2009.

LOZANO LEYVA, M., El fin de la ciencia, Ed. DEBOLS!LLO, 2013.

MELLADO RUIZ, L., “Obligación de uso de biocarburantes y biogás”, Mellado Ruiz, Lorenzo (coord.), Energías renovables, ahorro y eficiencia energética en Andalucía. Régimen jurídico, Atelier, 2012, pp. 83-163.

— "Bases teóricas y manifestaciones jurídico-sectoriales de los nuevos enfoques de sostenibilidad agrícola", Actualidad Jurídica Ambiental, ISSN-e 1989-5666, núm. 3 (junio), 2012, pp. 1-20.

NAVARRO RODRÍGUEZ, P., "La nueva Comisión Nacional de los Mercados y la Competencia: naturaleza, régimen jurídico e independencia”, Revista General de Derecho Administrativo, núm. 34, Iustel, 2013, pp. 1-52. 
PAREJO NAVAJAS, T., La estrategia territorial europea. La percepción comunitaria del uso del territorio, Marcial Pons, 2004.

PÉREZ GABALDÓN, M., La gestión intergubernamental de la politica de cambio climático en España, Aranzadi, 2013.

— Comentario jurisprudencial "Sentencia del Tribunal General (Sala Primera ampliada) de 13 de diciembre de 2013. (Asunto T-240/10). Hungría / Comisión. Publicada en el DO C 209, de 31.7.2010": $<$ http://www.actualidadjuridicaambiental.com//?s=Isabel+Hernández\&submit.x=14\&su bmit.y $=5 \&$ submit $=$ Search $>$

— "El papel de la ingeniería genética en el proceso de producción de bioetanol de segunda generación". Publicado por Javier Dufour el 28 marzo de 2014: <http://www.madrimasd.org/blogs/energiasalternativas/2014/03/28/132291>

— "La energía que viene del mar". En <http://www.jotdown.es/2014/05/la-energia-queviene-del-mar/>

\section{Recursos electrónicos y páginas web de interés:}

- Web del programa europeo de investigación Horizon 2020: $<$ http://ec.europa.eu/programmes/horizon2020/en>

- Portal español del Programa Marco de Investigación e Innovación de la UE. Gobierno de España. Ministerio de Economía y Competitividad: $<$ http://eshorizonte2020.es>

- Centro de Desarrollo de Energías Renovables (CEDER). Centro nacional para la investigación, el desarrollo y el fomento de las energías renovables. $<$ http://www.ceder.es/CEDERportal/>

- $\quad$ <http://www.abcburkina.net/es/nos-dossiers/vu-au-sud-vu-du-sud/857-444jatrophan-lnla-colere-des-paysansnr>.

— Consejo de la Unión Europea:

$<$ http://www.consilium.europa.eu/meetings?lang=es\&id=ccb4a244-88cd-41c8-8ee580b26ae8404d.> 
— Unión Europea: <http://europa.eu/index_es.htm>

- Ministerio de Industria, Energía y Turismo, Secretaría de Estado de Energía: $<$ http://www.minetur.gob.es/energia/es-

es/secretariadeestado/Paginas/SecretariaDeEstado.aspx>

- Comisión Nacional de la Energía (CNE), ya integrada en la Comisión Nacional de los Mercados y la Competencia (CNMC): <http://www.cne.es/cne/Home>; $<\mathrm{http}: / /$ www.cnmc.es $>$ 